\title{
Evaluation of Levetiracetam for Early Post- Traumatic Seizure Prophylaxis: A Level II Trauma Center Experience
}

\section{Timothy A. Amin}

Brooklyn Hospital Center

\section{Steven F. Nerenberg}

St. Joseph's University Medical Center

Osama A. Elsawy

St. Joseph's University Medical Center

Antai Wang

New Jersey Institute of Technology

Jackie P. Johnston ( $\nabla$ jackie.johnston@pharmacy.rutgers.edu )

St. Joseph's University Medical Center

\section{Research Article}

Keywords: Traumatic brain injury (TBI), Levetiracetam, Post-Traumatic Seizure Prophylaxis, Level II, Trauma Center Experience

Posted Date: December 6th, 2021

DOI: https://doi.org/10.21203/rs.3.rs-1075454/v1

License: (c) (i) This work is licensed under a Creative Commons Attribution 4.0 International License. Read Full License 


\section{Abstract}

Background: Traumatic brain injury (TBI) can induce early or late post-traumatic seizures (PTS). While PTS incidence is low, prophylaxis is used despite a lack of consensus on agent or duration.

Levetiracetam (LEV) for early PTS prophylaxis is preferred due to its safety and efficacy. The purpose of this study was to evaluate LEV for early PTS prophylaxis.

Methods: A single-center, retrospective chart review of TBI patients $\geq 18$ years who received LEV for early PTS prophylaxis between August 2018 - July 2019. The primary outcome was LEV duration. Secondary outcomes were incidence of seizure, intensive care unit (ICU) and hospital length of stay (LOS).

Results: Of the 137 included, mean age was $59 \pm 20$ years and $69.3 \%$ were male. The mean admission GCS was $13 \pm 4$ and $77.4 \%$ had mild TBI. Median LEV duration was 7 (IQR $4-10$ ) days and $13.9 \%$ met recommended 7-day duration. Those prescribed LEV $>7$ days had more than twice the median LEV duration than those prescribed $\leq 7$ days $(10.25$ (8.5-15.5) vs 4 (1.5-4.5) days, $p<0.0001)$. EEG-confirmed PTS occurred in $2.2 \%$, with an early PTS incidence of $0.73 \%$. Median ICU and hospital LOS were 2 (IQR 17) and 7 (IQR 3-16) days, respectively.

Conclusions: The incidence of PTS was low as most patients in our study had mild or moderate TBI. Early PTS prophylaxis with LEV for 7 days is appropriate, although the majority of patients did not meet the recommended duration. Efforts to standardize and implement PTS prophylaxis protocols are needed.

\section{Background}

Traumatic brain injury (TBI) can induce early ( $\leq 7$ days post-TBI) or late ( 7 days post-TBI) acute symptomatic seizures. ${ }^{1}$ Severity of TBI is commonly determined using the Glasgow Coma Score (GCS): mild (GCS 13-15), moderate (GCS 9-12), and severe (GCS < 9). ${ }^{1,2}$ Post-traumatic seizures (PTS) occur more often in severe TBI, meanwhile rates of subclinical seizures may be as high as $25 \%{ }^{3}$ The rate of post-traumatic epilepsy and recurrent seizures occurring more than 7 days post-TBI are significantly higher when compared to epilepsy rates in the general population. ${ }^{4,5}$ Risk factors for PTS include severe $\mathrm{TB}$, chronic alcohol use, prolonged post-traumatic amnesia, linear/depressed skull fracture, younger age (<65 years), subdural hematoma, intracerebral hematoma, penetrating head injury, and immediate seizures. ${ }^{6-8}$

Prophylaxis is routinely administered to prevent development of PTS, status epilepticus, chronic epilepsy, herniation, and death. ${ }^{4,7}$ While timely initiation reduces complications of early PTS (i.e., pneumonia, acute respiratory distress syndrome, acute renal failure, pulmonary embolism, and elevated intracranial pressure), limited durations to avoid adverse drug events should be considered. ${ }^{5} \mathrm{~A}$ randomized, doubleblind study of $404 \mathrm{TBI}$ patients comparing phenytoin (PHT) to placebo for one year, found that $\mathrm{PHY}$ reduces seizures during the first week following injury. ${ }^{9}$ Similar results were seen in a pooled analysis of 6 clinical trials of antiepileptic drugs (AED) (carbamazepine, phenobarbital, and PHT) of 1405 patients 
showing that AEDs reduced early, but not late PTS. ${ }^{10}$ Therefore, The Brain Trauma Foundation and American Association of Neurologic Surgeons recommend a 7-day duration for early PTS prophylaxis. ${ }^{3}$

While the duration of PTS prophylaxis is well defined, the choice of AED remains controversial. The Brain Trauma Foundation and American Association of Neurologic Surgeons recommend PHT for early PTS prophylaxis when "the overall benefit is felt to outweigh the complications associated with such treatment". ${ }^{3}$ Despite the inability to recommend LEV for PTS prophylaxis due to lack of sufficient evidence it is widely used. . $3,5,11$ A prospective, multicenter study of 813 consecutive blunt TBI patients, found LEV was not associated with differences in occurrence of early or late PTS, ADRs, or mortality when compared to PHT. ${ }^{12}$ An equivalence study of LEV and PHT in 32 severe TBI patients found no differences in Glasgow Outcome Scale at 3 and 6 months or seizures, although LEV was associated with an increased seizure tendency on electroencephalography (EEG). ${ }^{13}$ Similar results were seen in a prospective, randomized, single-blinded study finding no difference in seizure occurrence or death between LEV and PHT. Furthermore, LEV resulted in less undesirable side effects and better long-term outcomes for survivors. ${ }^{14}$ Thus, LEV is preferred for its well-established efficacy and lack of required therapeutic drug monitoring, tolerability, and relatively minimal drug-drug/drug-food interactions.

When considering LEV for early PTS prophylaxis, dosing 500-1000 mg every 12 hours intravenous or oral is initiated immediately following injury. Low-dose LEV $500 \mathrm{mg}$ every 12 hours has been shown to be effective in early PTS prevention and may be considered. ${ }^{15}$ While a 7-day duration is appropriate for early PTS prophylaxis, this practice is not widely agreed upon nor implemented. Due to a lack of consensus regarding definitive PTS prophylaxis agents, dosing, and duration, it is important to establish institutionspecific protocols. In this study, we evaluated compliance with these recommendations pertaining to the use of LEV for early PTS prophylaxis at a tertiary care, academic, level II trauma center.

\section{Methods}

This is a single-center, retrospective chart review of patients $\geq 18$ years of age presenting with TBI who received LEV for early PTS prophylaxis between August 1, 2018, and July 31, 2019, at St. Joseph's University Medical Center in Paterson, NJ. Those with a known hypersensitivity to LEV, pre-admission use of chronic AED, history of seizure disorder, or lack of documented LEV duration were excluded. Patients who left against medical advice or died within 7 days of admission were also excluded. Patient demographics, home medications, past medical history, social history, reason for admission, mechanism of traumatic brain injury, admission unit and in-hospital mortality were evaluated. Medical records were also reviewed for AED (drug, dose, frequency, and duration) ordered during hospitalization and EEG. This study was reviewed and approved by the St. Joseph's Health institutional review board (EX2019-22). All methods were carried out in accordance with institutional guidelines and regulations.

The primary outcome was duration of LEV for PTS prophylaxis. Duration was defined as the total number of days LEV was prescribed (loading and maintenance doses) and included in-hospital days plus the number of days prescribed at discharge. Duration prescribed at discharge was obtained from discharge 
medication reconciliation, physician discharge notes, and prescription fill histories. Secondary outcomes included incidence of EEG-confirmed seizure, hospital and intensive care unit (ICU) length of stay (LOS). Additional secondary outcomes evaluated were time to administration of first LEV dose, administration of LEV loading dose, LEV maintenance dose (milligrams; $\mathrm{mg}$ ) and frequency, and co-administration or transition to an alternative AED. All durations, time to initiation, and LOS were measured in calendar days. Subgroup analyses were performed to identify differences in LEV duration for TBI severity, ICU admission, ICU LOS ( 7 vs $\geq 7$ days), and hospital LOS.

Demographic factors and clinical characteristics were summarized with counts (percentages) for categorical variables, means and standard deviations for normally distributed continuous variables, and medians and interquartile ranges for non-normally distributed variables. Median data was compared using the Mann-Whitney $U$ test and nominal data was compared using the Chi-Square test. All analyses were performed with Microsoft Excel Tool Pak 2015. Significance was defined as p-value $\leq 0.05$.

\section{Results}

Of the 290 admissions evaluated, 137 met inclusion criteria. Each admission was included as a separate patient encounter. Reasons for exclusion were discontinuation of LEV following loading dose $(n=56)$, age $<18$ years $(n=34)$, prior use of AEDs $(n=17)$, lack of documented LEV duration $(n=16)$, left against medical advice $(n=16)$, mortality within 7 days of admission $(n=10)$, and miscellaneous reasons $(n=$ 4). The mean age was $59 \pm 20$ years, the majority male (69.3\%) and about half White $(50.4 \%)$. Most patients presented with mild (77.4\%) followed by severe (13.9\%) TBI. The mean admission GCS was $13 \pm 4$. The most common mechanisms of injury were fall $(64.2 \%)$ and motor vehicle collision $(22.6 \%)$. Three-quarters (78.8\%) were admitted to the ICU. Additional baseline demographics are shown in Table 1. 


\begin{tabular}{|c|c|}
\hline Male, $n(\%)$ & $95(69.3)$ \\
\hline Age (years), mean $\pm S D$ & $60 \pm 21$ \\
\hline Weight $(\mathrm{kg})$, mean \pm SD & $76.3 \pm 16.9$ \\
\hline $\mathrm{BMI}\left(\mathrm{kg} / \mathrm{m}^{2}\right)$, mean $\pm \mathrm{SD}$ & $26.5 \pm 5$ \\
\hline \multicolumn{2}{|l|}{ Race, n (\%) } \\
\hline White & $69(50.4)$ \\
\hline Latino & $28(20.4)$ \\
\hline Black & $19(13.9)$ \\
\hline Other/Mixed & $21(15.3)$ \\
\hline $\mathrm{SCr}$ at admission $(\mathrm{mg} / \mathrm{dL})$, mean $\pm \mathrm{SD}$ & $1.2 \pm 2.6$ \\
\hline $\mathrm{CrCl}$ at admission, mean $\pm \mathrm{SD}$ & $87.6 \pm 41.6$ \\
\hline Glasgow Coma Score (GCS) at admission, mean \pm SD & $13 \pm 4$ \\
\hline \multicolumn{2}{|l|}{ Severity of TBI, n (\%) } \\
\hline Mild (GCS 13-15) & $106(77.4)$ \\
\hline Moderate (GCS 9-12) & $12(8.8)$ \\
\hline Severe (GCS 3-8) & $19(13.9)$ \\
\hline \multicolumn{2}{|l|}{ Mechanism of TBI, n (\%) } \\
\hline Fall & $101(64.2)$ \\
\hline Motor vehicle accident & $31(22.6)$ \\
\hline Insult to head & $17(12.4)$ \\
\hline Other & $1(0.7)$ \\
\hline
\end{tabular}

The median duration of LEV was 7 (IQR 4-10) days. Only $13.9 \%$ of patients were prescribed LEV for exactly 7 days. Median LEV duration correlated with TBI severity: mild 7 (IQR 4-9.5), moderate 7.6 (IQR 6.5-9.5) and severe 9 (IQR 7-12.8) days. Severe TBI had longer median LEV duration when compared to mild-moderate TBI patients (7 (IQR 4.5-9.5) vs 9 (IQR 7-12.8) days, $p=0.055)$. Patients prescribed LEV $>7$ days had more than twice the median LEV duration than those prescribed $\leq 7$ days (10.3 (IQR 8.5-15.5) 
vs 4 (IQR 1.5-4.5) days, $\mathrm{p}<0.0001$ ). (Figure 1. Duration of LEV for PTS Prophylaxis) Patients with inhospital LEV duration $<7$ days were more likely to be discharged on LEV than those $>7$ days $(54.88 \%$ vs. $17.78 \%, p<0.0001)$.

Thirty-three patients (23.9\%) received EEG monitoring. Three patients (2.2\%) had EEG-confirmed PTS, with two $(1.46 \%)$ meeting criteria for late onset. One patient $(0.73 \%)$ progressed to status epilepticus, for whom several risk factors for early PTS (GCS $\leq 10$, intracerebral hematoma) and post-traumatic epilepsy (severe TBI and early PTS prior to discharge, acute intracerebral hematoma, and age $>65$ years) were noted.

A total of 107 patients required ICU admission with a median ICU LOS was 2 (IQR 1-7) days. Median LEV duration differed significantly among patients requiring ICU admission and those who did not (7.5 (IQR 4.5-10.5) vs 5.3 (IQR 2.3-8.3), $\mathrm{p}=0.0223$ ). There was no difference in LEV duration between ICU LOS $<7$ days versus $\geq 7$ days (7 (IQR 4.3-9.3) vs 7.5 (IQR 4-11.5), $\mathrm{p}=0.328$ ). Median hospital LOS was 7 (IQR 316) days. Nearly $50 \%$ had hospital LOS $<7$ days and 42 patients were discharged on LEV prior to hospital day 7. In-hospital LEV duration was longer when hospital LOS $>7$ days versus LOS $<7$ days [9 (IQR 712.9 ) vs. 2.8 (IQR 1.5-3.6) days, $\mathrm{p}<0.00001]$. Among patients discharged on LEV, those with a hospital LOS $>7$ days had prolonged total (in-hospital and outpatient post-discharge) duration of LEV when compared to those with LOS $<7$ days [11 (IQR 9-31.5) vs. 5.8 (IQR 4.6-10) days, $\mathrm{p}<0.05$ ). (Figure 2. Duration of Therapy for Patients Discharged on LEV) Approximately one-third (30.7\%) received a LEV loading dose, with a mean dose of $976 \pm 107.8 \mathrm{mg}$. The loading dose was administered in the emergency department (50\%) or the admitting unit (50\%) on the day of admission. The majority (93.4\%) of patients received LEV 500 mg twice daily, with therapy starting on hospital day $0(65 \%)$ or day $1(33.6 \%)$. Two patients (1.5\%) had an alternative AED ordered concurrently, while four (2.9\%) were switched from LEV to an alternative AED during hospitalization. (Table 2) 
Table 2

Secondary Outcomes

Received LEV LD, $\mathrm{n}(\%)$

LEV LD $(\mathrm{mg})$, mean \pm SD
$42(30.7)$

$976 \pm 107.8$

Location of LEV LD administration, $\mathrm{n}(\%)$

Emergency Department

In-patient unit

LEV MD (mg), mean \pm SD

$500 \pm 96.8$

Missed LEV MD (days), mean \pm SD

$0.3 \pm 0.7$

AED ordered concurrently, $\mathrm{n}(\%)$

Phenytoin

Valproic Acid

Switch from LEV to alternative AED, $n$ (\%)

$\mathrm{AED}=$ antiepileptic drug, IQR = interquartile range, $\mathrm{LD}=$ loading dose, $\mathrm{LEV}=$ levetiracetam, $\mathrm{MD}=$ maintenance dose, $\mathrm{SD}=$ standard deviation

\section{Discussion}

Incidence of early PTS varies with TBI severity, though prophylaxis in the first 7 days may reduce the risk of post-TBI seizure disorders. In this study, most participants received LEV $500 \mathrm{mg}$ twice daily for at least 7 days, starting on the day of admission. While this dosing scheme and time to initiation coincide with current recommendations, the total duration in our study did not. Factors contributing to longer duration of LEV therapy included ICU admission, longer hospital LOS, and higher TBI severity. While it is unclear why, in-hospital and total LEV duration were greater with longer hospital LOS. This may be due to disease severity or concern for seizures after day 7 , although only $13.9 \%$ of the study population presented with severe TBI. While not directly assessed, other potential reasons for LEV duration $>7$ days include prescriber specialty (critical care, neurosurgery, neurology), standardized AED duration of 30 days in computerized physician order entry system, and accidental renewals by ordering providers. Of note, twofifths of the study population were discharged on LEV with a median total duration of 7.5 days.

The incidence of PTS in our study is significantly lower than prior reports. This may be due to a smaller percent of patients presenting with severe TBI or lack of detection. Only one-quarter of patients had EEG, thus the potential for subclinical seizures cannot be ruled out. Our results indicate that for patients without a prior history of seizures, LEV monotherapy is sufficient. The decision to change to alternative AED or initiate a combination of AED should be made on a patient specific basis. 
There are several limitations to our study. First, this was a retrospective, single-center cohort study therefore all data collected was obtained through electronic medical records. As a result, differences in prescribing patterns among providers were not assessed. Second, despite documentation of LEV at discharge, we cannot exclude the possibility that patients may not have been continued on or may not have been adherent to therapy. Third, most patients included had mild TBI therefore our results may not represent PTS incidence for all TBI severities. Forth, in-hospital subclinical seizures were not evaluated in most patients. Use of EEG should be considered in patients with more severe TBI and risk factors for PTS. Lastly, our study did not evaluate for late PTS therefore the incidence may be under-reported.

\section{Conclusions}

Most patients in our study had mild or moderate TBI, therefore the incidence of PTS was low. Early PTS prophylaxis with LEV for 7 days is appropriate, although the majority of patients did not meet the recommended duration. Efforts to standardize and implement PTS prophylaxis protocols are needed. Furthermore, prospective, multi-center, randomized studies are needed to further evaluate LEV for PTS prophylaxis and the relation to TBI severity.

\section{Abbreviations}

AED = Antiepileptic drugs

EEG $=$ Electroencephalography

GCS = Glasgow Coma Score

$\mathrm{ICU}=$ Intensive care unit

$\mathrm{IQR}=$ Interquartile range

LEV = Levetiracetam

LOS = Length of stay

$M G=$ Milligrams

$\mathrm{PHT}=$ Phenytoin

PTS $=$ Post-traumatic seizures

$\mathrm{TBI}=$ Traumatic brain injury

\section{Declarations}


Ethics approval and consent to participate: This study was reviewed and approved by the St. Joseph's Health institutional review board (EX2019-22). The authors confirm that all experiments were performed in accordance with relevant guidelines and regulations.

Consent for publication: This study was granted exemption for informed consent. Need for informed consent was waived by the St. Joseph's Health ethics committee due to the retrospective nature of the study and less than minimal risk to study participants.

Availability of data and materials: The datasets used and/or analyzed during the current study are available from the corresponding author on reasonable request.

Competing interests: The authors declare that they have no competing interests.

Funding: The authors received no financial support for the research, authorship, or publication of the study.

Authors' contributions: TA, SF, OA and JJ contributed to the study design. TA obtained all data necessary for the study. TA, SF, AW, JJ analyzed and interpreted the data. TA, SF, OA and JJ contributed to the writing of the manuscript. All authors read and approved the final manuscript

Acknowledgements: Not applicable.

\section{References}

1. Yablon SA. Posttraumatic seizures. Arch Phys Med Rehabil. 1993 Sep;74(9):983-1001.

2. Sternbach GL. The Glasgow coma scale. J Emerg Med. 2000 Jul;19(1):67-71.

3. Brain Trauma Foundation; American Association of Neurological Surgeons; Congress of Neurological Surgeons: Guidelines for the management of severe traumatic brain injury. XIII. Antiseizure prophylaxis. J Neurotrauma. 2007, 24:83-86.

4. Ding K, Gupta PK, Diaz-Arrastia R. Epilepsy after Traumatic Brain Injury. In: Laskowitz D, Grant G, editors. Translational Research in Traumatic Brain Injury. Boca Raton (FL): CRC Press/Taylor and Francis Group; 2016. Chapter 14. Available from: https://www.ncbi.nlm.nih.gov/books/NBK326716/

5. Carney N, Totten AM, O'Reilly C, Ullman JS, Hawryluk GW, Bell MJ, et al. Guidelines for the Management of Severe Traumatic Brain Injury, Fourth Edition. Neurosurgery. 2017 Jan 1;80(1):6-15.

6. Xu T, Yu X, Ou S, Liu X, Yuan J, Huang H, et al. Risk factors for posttraumatic epilepsy: A systematic review and meta-analysis. Epilepsy Behav. 2017 Feb;67:1-6.

7. Torbic H, Forni AA, Anger KE, Degrado JR, Greenwood BC. Use of antiepileptics for seizure prophylaxis after traumatic brain injury. Am J Health Syst Pharm. 2013 May 1;70(9):759-66.

8. Ritter AC, Wagner AK, Fabio A, Pugh MJ, Walker WC, Szaflarski JP, et al. Incidence and risk factors of posttraumatic seizures following traumatic brain injury: A Traumatic Brain Injury Model Systems Study. Epilepsia. 2016 Dec;57(12):1968-1977. 
9. Temkin NR, Dikmen SS, Wilensky AJ, Keihm J, Chabal S, Winn HR. A randomized, double-blind study of phenytoin for the prevention of post-traumatic seizures. N Engl J Med. 1990 Aug 23;323(8):497502 .

10. Hazama A, Ziechmann R, Arul M, Krishnamurthy S, Galgano M, Chin LS. The Effect of Keppra Prophylaxis on the Incidence of Early Onset, Post-traumatic Brain Injury Seizures. Cureus. 2018;10(5):e2674.

11. Schierhout G, Roberts I. Prophylactic antiepileptic agents after head injury: a systematic review. J Neurol Neurosurg Psychiatry. 1998;64(1):108-112.

12. Inaba K, Menaker J, Branco BC, Gooch J, Okoye OT, Herrold J, Scalea TM, Dubose J, Demetriades D. A prospective multicenter comparison of levetiracetam versus phenytoin for early posttraumatic seizure prophylaxis. J Trauma Acute Care Surg. 2013 Mar;74(3):766-71; discussion 771-3.

13. Jones KE, Puccio AM, Harshman KJ, Falcione B, Benedict N, Jankowitz BT, et al. Levetiracetam versus phenytoin for seizure prophylaxis in severe traumatic brain injury. Neurosurg Focus. 2008 Oct;25(4):E3.

14. Szaflarski JP, Sangha KS, Lindsell CJ, Shutter LA. Prospective, randomized, single-blinded comparative trial of intravenous levetiracetam versus phenytoin for seizure prophylaxis. Neurocrit Care. 2010 Apr;12(2):165-72.

15. Patanwala AE, Kurita A, Truong E. Low-dose levetiracetam for seizure prophylaxis after traumatic brain injury. Brain Inj. 2016;30(2):156-8.

\section{Figures}

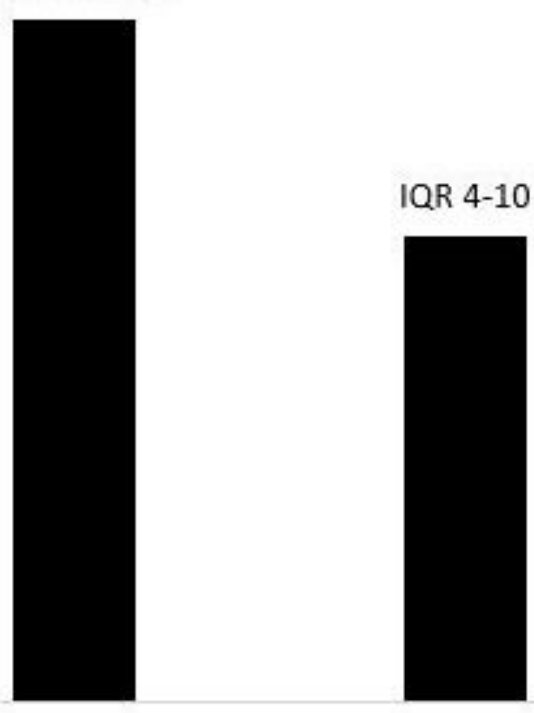

LEV $<7$ days $(n=52$ LEV $=7$ days $(n=19)$

LEV > 7 days $(n=66)$

All patients ( $n=137)$ 
Median LEV duration measured in calendar days were compared among all patients, those with durations $<7,=7$ days or $>7$ days. All patients had a median LEV duration of 7 days. Of those with LEV durations $>$ 7 days, total median LEV duration was 10.3 days.

12

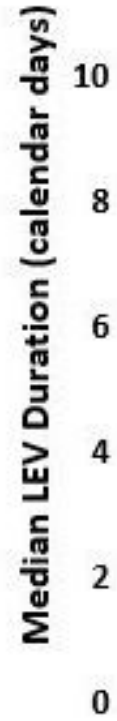

IQR $4.6-10$

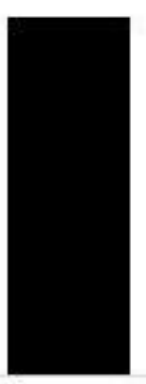

Hospital LOS $<7$ days $(n=42)$

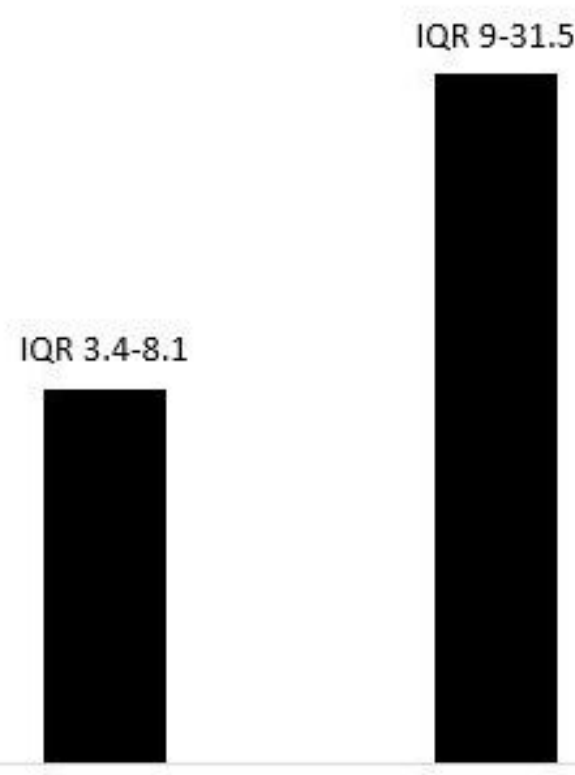

Hospital LOS $=7$ days $(n=4)$
Hospital LOS > 7 days $(n=9)$
IQR 6-10.3

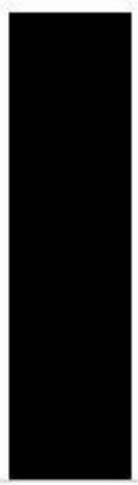

All patients $(n=55)$

\section{Figure 2}

Median LEV duration measured in calendar days were compared among all patients, those with hospital LOS $<7$ days, $=7$ day and $>7$ days. Of those who had hospital LOS $>7$ days, LEV duration was approximately twice as long compared to those with hospital LOS $<$ or $=7$ days.

\section{Supplementary Files}

This is a list of supplementary files associated with this preprint. Click to download.

- SupplementalFigure1.PatientEnrollment.jpg 